\title{
New Off-Board Solution for Predicting Vehicles' Intentions in the Highway On-Ramp Using Probabilistic Classifiers (Student Abstract)
}

\author{
Zine el abidine Kherroubi \\ Groupe Renault \\ Guyancourt, 78280 \\ zine-el-abidine.z.kherroubi@renault.com
}

\author{
Samir Aknine \\ Claude Bernard Lyon 1 University \\ Villeurbanne, 69100 \\ samir.aknine@univ-lyon1.fr
}

\author{
Rebiha Bacha \\ Groupe Renault \\ Guyancourt, 78280 \\ rebiha.bacha@ renault.com
}

\begin{abstract}
This paper proposes a new approach for predicting drivers' intentions in a Highway on-ramp merge situation using a central road side unit (RSU) with probabilistic classifiers.
\end{abstract}

\section{Introduction and Model}

Most current autonomous driving systems are perception based, and rely on a myriad of on-board sensors. Sharing these on-board data would be beneficial to other vehicles on the road. The present work is part of an industrial research project for a car manufacturer. The first objective of this work is to design a strategy that predicts drivers' intentions in the highway on-ramp merge situation. We considered this use case first because the highway on-ramp merge is one of the situations requiring considerable efforts from the driver, consisting in recognizing surrounding vehicles.

A directed graph model to estimate drivers' behaviors and decisions in the highway on-ramp merge situation is proposed. The output of this model is compared using several probabilistic classifiers. The classical logistic regression (LRM) was used as a discriminative classifier ( $\mathrm{Ng}$ and Jordan 2001)(Hosmer and Lemeshow 2005). The Naïve-Bayes model (NB) (cf. Figure 1) and two of its variants (the Tree Augmented Naïve-Bayes (TAN) (cf. Figure 2) and the General Bayesian Network (GBN) (cf. Figure 3)) were used as generative classifiers (Friedman, Geiger, and Goldszmidt 1997). Also, the k-nearest neighbors classifier (KNN) and the Artificial Neural Network (ANN) were included in this comparison. The results show that the LRM, KNN and ANN classifiers give better prediction performances than the generative classifiers NB, TAN and GBN.

The model structure is composed of three layers: the vector $X$ which contains the vehicle data (mainly dynamic data), the vector $C$ which contains the vehicle situation context, and finally the output $I$ which is the intention of merging or not merging for vehicle.

Vector X: contains the vehicle states: $\{$ Position, Speed, Acceleration $\}$.

Vector $C$ : contains the features of the local situation context, and contains for the vehicle in the merge lane (resp.

Copyright (C) 2020, Association for the Advancement of Artificial Intelligence (www.aaai.org). All rights reserved. main lane): $C_{1}$ (resp. $C_{1}^{\prime}$ ): Distance from the merging point, $C_{2}$ (resp. $C_{2}^{\prime}$ ): Speed, $C_{3}$ (resp. $C_{3}^{\prime}$ ): Acceleration, $C_{4}$ (resp. $\left.C_{4}^{\prime}\right)$ : Relative distance between the vehicle in the main lane and the vehicle in the merge lane, $C_{5}$ (resp. $C_{5}^{\prime}$ ): Relative speed between the vehicle in the main lane and the vehicle in the merge lane, $C_{6}$ (resp. $C_{6}^{\prime}$ ): Relative acceleration between the vehicle in the main lane and the vehicle in the merge lane, $C_{7}$ (resp. $C_{7}^{\prime}$ ): Relative distance from the vehicle above the merging point in the main lane, $C_{8}$ (resp. $C_{8}^{\prime}$ ): Relative speed from the vehicle above the merging point in the main lane, $C_{9}$ (resp. $C_{9}^{\prime}$ ): Relative acceleration from the vehicle above the merging point in the main lane.

Vector I: contains the intention of merging or not merging. The probability of merging is deduced from the situation context vector $P(I / C)$. An output probability with a value close to 1 means that the vehicle has the intention to merge before the vehicle in the other lane (either main lane or merge lane).
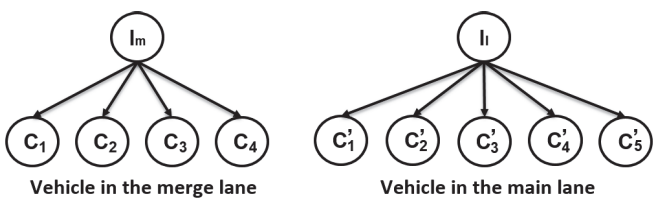

Figure 1: Naïve-Bayes structure example.
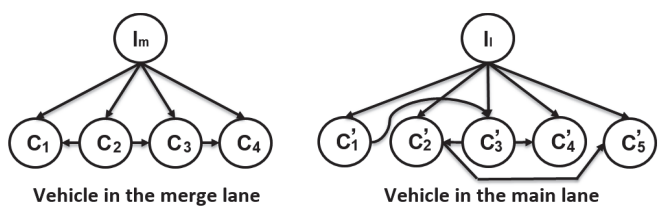

Figure 2: Tree Augmented Naïve-Bayes structure example. 

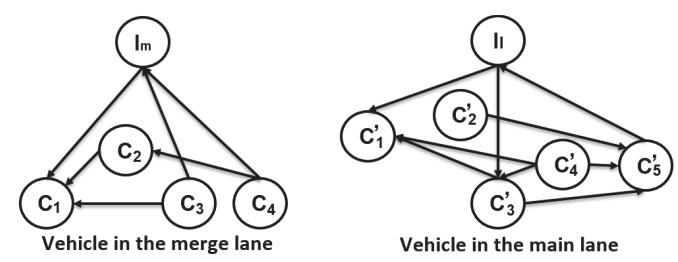

Figure 3: General Bayesian Network structure example.

\section{Experimental evaluation}

The proposed model was simulated using the Next Generation Simulation (NGSIM) (Cambridge Systematics 2005). The data used to train our model correspond to vehicles' trajectories on a segment of interstate 80 in Emeryville (San Francisco), California collected between 4:00 p.m. and 4:15 p.m. on April 13, 2005. We notice that the discriminative

\begin{tabular}{|c|c|c|c|c|}
\hline Classifier & Accuracy & Precision & Recall & F1 Score \\
\hline LRM & $99 \%$ & $99 \%$ & $99 \%$ & $99 \%$ \\
\hline NB & $92 \%$ & $91 \%$ & $90 \%$ & $91 \%$ \\
\hline TAN & $98 \%$ & $98 \%$ & $97 \%$ & $98 \%$ \\
\hline GBN & $97 \%$ & $96 \%$ & $96 \%$ & $96 \%$ \\
\hline KNN & $96 \%$ & $97 \%$ & $94 \%$ & $95 \%$ \\
\hline ANN & $98 \%$ & $99 \%$ & $98 \%$ & $98 \%$ \\
\hline
\end{tabular}

Table 1: Models' performances.

classifier LRM and the ANN give the best performances over the generative classifiers (NB, TAN and GBN) and the KNN classifier. In fact, the precision for the LRM and ANN are $99 \%$. In contrast to the LRM classifier, the ANN classifier can have different structures. In fact, accuracy and precision were around $96 \%$ when we previously used only 12 nodes in the hidden layer and 6 nodes in the output layer. Concerning the Recall criteria, both the LRM and ANN show good values. This means that in all the situations where the vehicle in the merge lane takes priority and merges before the main lane vehicle, we are able to predict the majority of these situations accurately, which will be very useful for the decision-making strategy. Finally, the F1 score takes both FALSE positives and FALSE negatives into account. The positive prediction refers to the intention to merge for the vehicle in the merge lane (model output 1), while the negative prediction refers to the vehicle intention to pass for the main lane (model output 0 ).

In order to verify the feasibility of a centralized off-board solution, we calculate the time to arrival to the merging point for the vehicle in the merge lane $\left(T_{m}\right)$, and the time to arrival to the merging point for the vehicle in the main lane $\left(T_{l}\right)$ at the instant when the model predicts the first True intention of the vehicle (cf. Figure 4). We estimate that the decisionmaking for connected and autonomous vehicles needs a response time of $T R=0.5 \mathrm{sec}$ :

- $0.4 \mathrm{sec}$, for the vehicle response time (Armand 2016).

- $0.1 \mathrm{sec}$, for the communication latency (ETSI 2014).

The values of the time to arrival for the first True prediction are shown in Figure 4. For each one of the classifiers, we

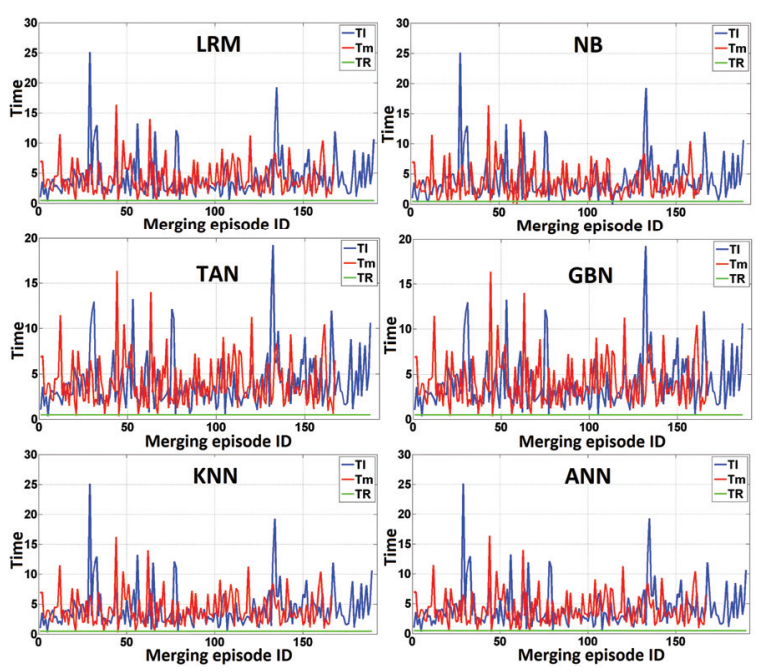

Figure 4: Time to arrival for the first True prediction

notice that the model predicts the intention of the vehicles before they reach the on-ramp merge point by a mean of 3.96 $\mathrm{sec}$ for the vehicle in the merge lane, and $4.08 \mathrm{sec}$ for the vehicle in the main lane, which we estimate to be sufficient for decision-making.

\section{Conclusion}

In this paper, we proposed a novel solution to predict drivers' intentions in a highway on-ramp merge situation using probabilistic classifiers and a central road side unit that uses communication and sensors. The model was validated using real-world data and the 5-fold cross-validation with existing probabilistic classifiers. The performance metrics show the best values for the LRM and ANN classifiers, which yield an accuracy and precision around $99 \%$. The model is robust for predicting both the main lane vehicles' and the merge lane vehicles' intentions.

\section{References}

Armand, A. 2016. Situation understanding and risk assessment framework for preventive driver assistance. Ph.D. Dissertation, University of Paris-Saclay.

Cambridge Systematics, I. 2005. Ngsim i-80 data analysis (4:00 p.m. to 4:15 p.m.). Technical report, Federal Highway Administration.

ETSI. 2014. Vehicular communications : Basic set of applications. part 2: Specification of cooperative awareness basic service. Technical report, Intelligent Transport Systems.

Friedman, N.; Geiger, D.; and Goldszmidt, M. 1997. Bayesian network classifiers. Machine Learning, 29.

Hosmer, D. W., and Lemeshow, S. 2005. Applied Logistic Regression. Wiley series in Probability and Statistics.

Ng, A. Y., and Jordan, M. I. 2001. Discriminative vs. generative classifiers: A comparison of logistic regression and naive bayes. Advances in Neural Information Processing Systems 14: 841-848. 